\title{
Hypersensitivity reactions to metal implants: laboratory options
}

Anna Maria Carossino, Christian Carulli, Simone Ciuffi, Roberto Carossino, Giorgia Donata Zappoli Thyrion, Roberto Zonefrati, Massimo Innocenti and Maria Luisa Brandi

\begin{abstract}
Background: All implant compounds undergo an electrochemical process when in contact with biological fluids, as well as mechanical corrosion due to abrasive wear, with production of metal debris that may inhibit repair processes. None of the commonly-used methods can diagnose implant allergies when used singly, therefore a panel of tests should be performed on allergic patients as pre-operative screening, or when a postoperative metal sensitisation is suspected.
\end{abstract}

Methods: We analysed patients with painful prostheses and subjects prone to allergies using the Patch Test in comparison with the Lymphocyte Transformation Test. Cytokine production was evaluated to identify prognostic markers for early diagnosis of aseptic loosening. Metal debris endocytosis and cytoskeletal rearrangement was visualised by confocal microscopy.

Results: Our results demonstrate that the Lymphocyte Transformation Test can identify patients who have a predisposition to develop allergic reactions and can confirm the diagnosis of hypersensitivity in patients with painful prostheses.

The prevalence of a Th2-cytokine pattern may be used to identify predisposition to the development of allergic diseases, while the selective presence of osteoclastogenic cytokines may be used as predictor of a negative outcome in patients with painful prosthesis.

The hypothesis of the prognostic value of these cytokines as early markers of aseptic loosening is attractive, but its confirmation would require extensive testing.

Conclusions: The Lymphocyte Transformation Test is the most suitable method for testing systemic allergies. We suggest that the combined use of the Patch Test and the Lymphocyte Transformation Test, associated with cytokine detection in selected patients, could provide a useful tool for preventive evaluation of immune reactivity in patients undergoing primary joint replacement surgery, and for clinical monitoring of the possible onset of a metal sensitization in patients with implanted devices.

Keywords: Knee arthroplasty, Metal sensitivity, Lymphocyte transformation test, Patch test, Cytokines

\section{Background}

Total Knee Arthroplasty (TKA) is one of the most successful orthopaedic procedures for the treatment of osteoarthritis $[1,2]$. Particularly high rates of patient satisfaction have been achieved in recent decades thanks to the significant improvement in both surgical technique, implant design, and the characteristics of biomaterials. An increasing number of TKAs have been performed

\footnotetext{
* Correspondence: marialuisa.brandi@unifi.it

Department of Surgery and Translational Medicine, University of Florence, Viale Pieraccini 6, 50139 Florence, Italy
}

worldwide during recent years as a consequence of the aging population and a higher incidence of secondary osteoarthritis in younger patients [3].

Given the high percentage of hypersensitivity to metals (up to $10 \%$ ), particularly to nickel, in the general population, and the presence of this particular substance in standard knee implants [4], it may be crucial to verify the patient's hypersensitivity prior to surgery, in order to prevent reactions. This approach is further justified by the fact that a significant number of failures are expected to occur over the next few decades due to various 
emerging causes, such as patellar clunk syndrome, periprosthetic fractures, and hypersensitivity to metals [5].

Orthopaedic devices are generally well tolerated, but may sometimes generate corrosion products which cause periprosthetic bone resorption; this can lead to implant looseness and a second surgical procedure may be required to fix or replace the failed implant $[6,7]$.

The presence of small amounts of debris that can be removed through catabolic processes is consistent with biological tolerance of implants $[8,9]$, while high concentrations of free metal ions may be accumulated in the surrounding tissue, or carried through the bloodstream to distant organs $[10,11]$. Metal particles bind to serum proteins to form hapten-like complexes that may be identified by the immune system as antigens, and can activate local or systemic inflammatory reactions by recruiting macrophages, fibroblasts, lymphocytes, and osteoclasts, which induce proinflammatory and osteoclastogenic cytokine release [12]. This response, classified as a type-IV delayed hypersensitivity reaction, is mediated by antigen-presenting cells and $\mathrm{T}$ lymphocytes, and can occur either in the postoperative period or months and even years later $[7,13]$.

$\mathrm{T}$ cell activation produces a self-perpetuating loop whereby macrophages are recruited and activated, these in their turn present a class II major histocompatibility complex (MHC II) which can activate other sensitised T cells, and so on. Soluble ions and metal particles may induce monocyte/macrophage activation that stimulates the inflammatory response by various mechanisms. Particles can be internalised by phagocytosis, which is traditionally associated with the expression of MHC II on macrophages, while soluble ions can penetrate cells via passive or active transport.

Several studies have demonstrated that cell response may be affected by the size, shape, quantity and composition of the debris, and have suggested a possible correlation between the presence of corrosion products and the symptoms of localized or systemic allergic dermatitis $[14,15]$. Nevertheless, there is no evidence of a direct link, since immune response changes in relation to the general state of health, genetic susceptibility, and individual hypersensitivity [16].

The incidence of serious adverse reactions requiring implant removal is quite low, but the percentage of patients with postoperative local inflammatory symptoms, persistent pain, and poorly- functioning implants is not negligible, and demands thorough evaluation. Because the immune-related mechanisms of metal allergy development are not well understood, and several factors may contribute to overaggressive responses, this phenomenon may be underrated in the evaluation of persistent postsurgical pain.

To date, no in vivo and in vitro methods have been specifically assessed for their efficiency in testing allergies caused by implants. Patch testing (PT) tends to be used as the standard test because of its cost-effectiveness and technical simplicity, but its specific diagnostic usefulness is controversial. In fact, there is a remarkable difference between the immunological reaction deriving from antigen-presenting cells located in the skin layers and the systemic response to metal debris, which is mediated by macrophages and dendritic cells located in the periprosthetic area. Moreover, screening is not recommended because hapten exposure may itself induce sensitization. In addition, visual scoring of skin reactions is conditioned by the physician's experience and may be influenced by medications, the quality of the substances, and the time of reading $[17,18]$.

More specific laboratory methods, based on lymphocyte proliferation and measurement of cytokine release, have been proposed: the Lymphocyte Transformation Test (LTT), which evaluates the proliferative response of activated $\mathrm{T}$ lymphocytes, is based on evidence that allergic individuals produce memory $\mathrm{T}$ cells which are able to be activated by antigen exposure. Moreover, the detection of specific cytokines secreted as the result of an immune response indicates the qualitative and quantitative involvement of different cell types $[19,20]$.

Generally, the diagnosis of metal allergy in patients who have undergone a second surgical procedure due to the negative outcome of the first one is made by exclusion criteria: lack of evidence of infections, non-union, and mechanical failure. When an aseptic loosening is confirmed, revision surgery is the only option. In addition to the patient's suffering, the complexity and timeconsuming aspects of the revision procedures, as well as the related social and economic costs must all also be taken into account. It is thus useful, in order to choose the correct implant, to identify which metal is inducing the allergic reactions, [21, 22] both in allergic subjects and in patients with persistent post-surgical pain. TKA offers a unique model of study of such condition because it is the only procedures that may be performed using actual fully non-allergic implants.

\section{Purpose of this study}

We selected a group of patients with a clinical history of metal allergies who had undergone primary Total Knee Arthroplasty (pTKA), and a second group of patients with painful prostheses. Each patient was compared to a group of control subjects.

The aim of the study was to assess the ability of LTT and PT to discriminate between skin and systemic reactions. Cytokine production was evaluated to identify prognostic markers for early diagnosis of aseptic loosening. Finally, to assess metal endocytosis and cytoskeletal rearrangement, cell interaction with metal particles was visualised by confocal microscopy. 


\section{Methods}

\section{Patients}

Thirty potentially allergic patients, divided into two groups, were studied. Demographic and clinical data were collected using a questionnaire. Group 1 included eight patients scheduled for pTKA, who had a documented clinical history of metal allergy and hypersensitivity reactions (eczematous rashes, rhinitis, asthma). Group 2 was composed of twenty-two patients with a painful TKA. These were evaluated for the nature, location, onset and duration of their pain, at least 6 months postoperatively, and their pain was classified on the basis of a Visual Analogical Scale $\geq 7$. All signs of the common causes of failure (infection, instability) were ruled out. Eleven of the patients referred metal allergies (Group 2A), while the other eleven did not refer any allergies and had no clinical signs of sensitisation (Group 2B).

All patients were studied by medical history, blood tests for infection indices (hemochrome, CRP, ESR) and radiological evaluation (anterior-posterior, lateral and patellar views, long-standing radiograms, CT scan for rotational evaluation of components). Functional limitation was measured using a Range of Motion (ROM) evaluation, and by the Knee Society Score (KSS) and the Western Ontario and McMaster Universities Osteoarthritis Index (WOMAC).

Controls (Group 3) included nine volunteers unaffected by skin disorders or immunological, metabolic, or chronic diseases, and without any previous known contact with metal implants.

\section{Patch test}

All subjects were tested according to the guidelines suggested by the Società Italiana di Dermatologia Allergologica Professionale e Ambientale (SIDAPA) based on international guidelines $[23,24]$.

Metal allergy was tested by using the following haptens: Cobalt Chloride 1\%, Nickel Sulphate 5\%, Potassium Dichromate $0.5 \%$, and Chromium III 2\%. (F.I.R.M.A SpA, Florence, Italy). Vaseline, used as vehicle for patch test, was assayed as a negative control.

A drop of each hapten was smeared on Haye's chamber test, which was applied to areas of the left side of the patient's upper back that were free of erythema and dermatitis.

The reading was performed after 48 and $72 \mathrm{~h}$, and results were recorded based on the second reading. According to the recommendation from the International Contact Dermatitis Research Group, allergic responses from $1+$ to $3+$ were interpreted as a positive reaction, and were scored as: 1+ (week non-vesicular erythema with edema and infiltration), 2+ (moderate homogeneous redness, with edema, infiltration and vesicles), and $3+$ (strong homogeneous redness, infiltration and bullous reaction).
A negative reading ( 0$)$ or a doubtful reaction (+?, only erythema without infiltration) was interpreted as a negative response [25].

\section{Lymphocyte transformation test}

Samples were collected after informed consent and before PT, to prevent sensitisation. Peripheral Blood Mononuclear Cells (PBMC) were isolated with Lymphocyte Separation Medium and resuspended in RPMI-1640 containing 10\% Fetal Bovine Serum. Non-toxic concentrations of challenge metals $(0,1 \mathrm{mM} ; 0,01 \mathrm{mM})$ were selected by a dose response curve. $2 \times 10^{5}$ cells/well were seeded in triplicate in 96-well plates with or without Chromium (III) chloride $\left(\mathrm{CrCl}_{3}\right)$, Chromium powder (Cr), Nickel (II) chloride $\left(\mathrm{NiCl}_{2}\right)$, Nickel nanopowder $(\mathrm{Ni})$, Cobalt powder (Co), Titanium powder $(\mathrm{Ti})$, and Molybdenum nanopowder (Mo). $\mathrm{Ni}$ and $\mathrm{Cr}$, the main sensitiser metals, were analyzed both in soluble and particulate form. Phytohemagglutinin (PHA 0,01 mg/ml) was used as control. After 5 days, cells were pulsed overnight with ${ }^{3} \mathrm{H}$-thymidine $(1 \mu \mathrm{Ci} /$ well $)$ and proliferation was assessed by scintillation counting. Results were expressed as Stimulation Index (SI = mean cpm-treated /mean cpmuntreated cultures). Culture media and supplements were purchased from Biowhittaker, (Lonza, Treviglio, Italy), chemicals from Sigma Aldrich (Milan, Italy).

\section{Luminex cytokine assays}

Cytokine production was evaluated in eight Group 1 patients, four Group 2A patients (all positive to LTT) and five Controls. Supernatants from metal-challenge PBMC were collected on day 5 and stored at $-80{ }^{\circ} \mathrm{C}$. Luminex multiplex array (Bio-Rad Laboratories, Hercules, CA, USA) was used to quantify: IL-1 $\beta$, IL-1ra, IL-2, IL-4, IL5, IL-6, IL-7, IL-8, IL-9, IL-10, IL-12, IL-13, IL-15, IL-17, eotaxin, bFGF, G-CSF, GM-CSF, IFN- $\gamma$, IP-10, MCP-1, MIP-1 $\alpha$, MIP-1 $\beta$, PDGF-BB, RANTES, TNF- $\alpha$, VEGF. Samples were analysed in duplicate using the reagents and the protocol supplied in the kit.

Out-of-range values (above or below detection limits) were rated as the highest and the lowest detectable concentrations. Cytokine production was expressed as Stimulation Index (SI = value of stimulated cultures/ value of unstimulated cultures).

Phase-contrast and laser scan confocal microscopy (LSCM) PBMC $\left(2 \times 10^{4}\right.$ cells/well $)$ were cultured in LabTek chamber-slides (Thermo Scientific-NUNC, Milan, Italy) for 5 days with or without $0.1 \mathrm{mM}$ and $0.01 \mathrm{mM}$ of selected metals. Reagents were purchased from Sigma Aldrich Milan, Italy. Cells were fixed with $4 \%$ paraformaldehyde, permeabilised in $0.2 \%$ Triton $\mathrm{X}-100$, and non-specific binding sites blocked using $2 \%$ Bovine Serum Albumin. To evaluate the cytoskeletal rearrangement of F- 
actin filaments, samples were stained with Phalloidin TRITC-conjugate. Nuclei were counterstained with Hoechst 33258. Samples were mounted in polyvinyl alcohol mounting medium with anti-fading DABCO and examined under an Axiovert $200 \mathrm{M}$ inverted LSM510 (Carl Zeiss, Oberkochen, Germany). Quantitative assessment of cells with internalized metals was carried out by counting a total number of 200 cells/ well, per metal treatment. Quantification of positive cells was expressed as the percentage fraction of total cell numbers counted for each metal.

\section{Statistical analysis}

The mean values of SI in allergic patients labelled for TKA and in patients with painful prostheses were compared to those of control subjects. The differences among the groups were evaluated using the ANOVA procedure (STATA statistical package, Stata Corp. 2009 - Stata Statistical Software: Release 10. College Station, TX: Stata Corp LP), and the REGRESS post estimation command was used to calculate the $p$-values of the differences between each group of patients (allergic, painful prosthesis) and control subjects.

Previous literature was analysed to perform a power calculation. Since several studies did not report effect size statistics, a raw estimate of an effect size was achieved using nonparametric statistics reported in conditions which were as similar as possible to ours [26].

The agreement between PT and LTT was estimated in absolute percentage and by means of the Kappa Statistic $(0-0,20=$ poor; $0,21-0,40=$ fair; $0,41-0,60=$ moderate; 0,61 0,80 = good; $0,80-1=$ very good), which takes chance agreement into account [27].

\section{Results}

Considering the relatively small sample size, we tried to perform a power calculation by analysing previous literature. Several studies did not report effect size statistics and often did not provide enough data to compute them. The raw estimate is between .49 and .64. In our case, with this effect size, comparing the 4 groups with ANOVA, the power is between 71 and $91 \%$.

\section{Patch test and lymphocyte reactivity}

Since none of the laboratory tests are able to provide certain diagnoses of allergy, we tried to correlate PT with the LTT. PT was evaluated according to the clinical scoring criteria of the International Contact Dermatitis Research Group [25].

The LTT was considered positive for SI $>2$. The enhanced proliferation was explained as the expression of a metal-specific lymphocyte response. All control subjects were found to be negative to PT as well as to the LTT. The demographic and clinical characteristics of the
30 patients, and their individual reactivity to $\mathrm{PT}$ and the LTT, are summarised in Table 1 . The two tests matched in $60 \%$ of cases. A complete correspondence (PT-LTTor $\mathrm{PT}+\mathrm{LTT}+$ ) for the same metals was achieved in $37 \%$ of tests. Contradictory results (PT + LTT-) were obtained in $13 \%$ of cases, while (PT-LTT+) in $17 \%$. Doubtful PT (10\%) were ruled out by the LTT (7\% negative and 3\% positive responses). The PT and LTT results obtained for each group of patients are detailed in Table 2.

The observed agreement between the two tests calculated by the Kappa Statistic, not considering PT as a gold standard, was $76.6 \%$ for all metals tested, with a Kappa value of 0.3 (fairly good agreement). Similar results were obtained by comparing the two tests for each metal.

Figure 1a,b shows the effect of sensitiser metals on lymphocyte response. Mean SI values for each of the 3 patient groups were compared to controls. At the concentration of $0.1 \mathrm{mM}$, (Fig. 1a), $\mathrm{NiCl}_{2}$ produced the highest degree of proliferative response, with a more than fivefold increase in Group 1 and Group 2A (5.1 and 5.3 respectively), and a twofold increase in Group 2B. Statistically significant difference was reached in Group $1(p=0.004)$ and Group 2A $(p=0.001)$. Moreover, $\mathrm{Ni}$ increased proliferative response by about twofold in Group 1 and Group 2A (2.2 and 1.7 respectively), with a statistically significant difference in Group $1(p=0.001)$, and Group 2A $(p=0.01)$. Group I patients showed a statistically significant proliferative response in the presence of $\mathrm{CrCl}_{3}(1.7 ; p=0.001)$ and $\mathrm{Cr}(1.4 ; p=0.03)$, while Group 2A patients showed a high proliferative response, about a sixfold increase, in the presence of Co $(5.7 ; p<0.0001)$.

At a concentration of $0.01 \mathrm{mM}$ (Fig. $1 \mathrm{~b}$ ), $\mathrm{CrCl}_{3} \mathrm{Cr}$ and $\mathrm{Ni}$ did not produce statistically significant responses. In the presence of $\mathrm{NiCl}_{2}$, Group 1 and Group $2 \mathrm{~A}$ showed about a twofold increase in proliferative response $(2 ; p=0.01$ and 2,$1 ; p=0.003)$. Co increased proliferative response to a lesser extent $(1.7 ; p=0.01)$. $\mathrm{Ti}$ and Mo did not produce statistically significant proliferative effects, remaining constantly below the threshold value of $\mathrm{SI}<2$. No significant differences were detected between the two concentrations of sensitiser metals.

\section{Luminex cytokine assays}

Although a wide range and sometimes large amounts of cytokines were produced, only some cytokines reached statistically significant levels, owing to the low number of subjects analysed (Fig. 2a-f).

The ratio of stimulated to unstimulated cultures in cytokine production was expressed as SI and averaged. Statistically significant differences were obtained comparing each of the two patient groups with controls. 
Table 1 Overview of demographic and clinical characteristics, and results of PT and LTT

\begin{tabular}{|c|c|c|c|c|c|c|c|c|c|}
\hline Group 1 & Gen & Dental fillings & $\begin{array}{l}\text { Familiarity } \\
\text { for allergy }\end{array}$ & $\begin{array}{l}\text { Professional } \\
\text { exposure }\end{array}$ & Known allergies & Jewelry allergy & Cosmetic allergy & PT reactivity & LTT response \\
\hline 1 & $F$ & Yes & None & None & $\mathrm{Ni}$ & Yes & None & $++++\mathrm{Ni}$ & $++++\mathrm{Ni}_{1}+\mathrm{Cr}_{1}$ \\
\hline 2 & $F$ & Yes & Yes & None & $\mathrm{Ni}$ & Yes & Yes & $++\mathrm{Ni}$ & $+++\mathrm{Ni},+\mathrm{Cr}_{1}+\mathrm{Ti}$ \\
\hline 3 & $F$ & Yes & None & None & $\mathrm{Ni}$ & Yes & None & $++\mathrm{Ni},+\mathrm{Co}$ & $+\mathrm{Ni}$ \\
\hline 4 & $F$ & Yes & Yes & None & $\mathrm{Ni}$ & Yes & None & $+++\mathrm{Ni}$ & $+\mathrm{Ni},+\mathrm{Cr}$ \\
\hline 5 & $F$ & Yes & None & None & $\mathrm{Ni}, \mathrm{Cr}$ & Yes & None & $+\mathrm{Ni}$ & Neg \\
\hline 6 & $F$ & Yes & None & Yes & $\mathrm{Ni}$ & Yes & Yes & $++\mathrm{Ni}$ & Neg \\
\hline 7 & $F$ & Yes & None & None & None & Yes & Yes & $+\mathrm{Ni}$ & $+\mathrm{Ni}$ \\
\hline 8 & $F$ & Yes & None & None & None & Yes & Yes & $+/-\mathrm{Ni}$ & $+\mathrm{Ni}$ \\
\hline \multicolumn{10}{|c|}{ Group 2A } \\
\hline 1 & $F$ & None & None & None & $\mathrm{Ni}$ & None & None & $++\mathrm{Ni}$ & $+\mathrm{Ni}$ \\
\hline 2 & $F$ & Yes & None & None & None & None & None & $++\mathrm{Co}$ & $+\mathrm{Ni},+\mathrm{Cr}_{1}+\mathrm{Mo}$ \\
\hline 3 & $F$ & None & None & Yes & $\mathrm{Ni}$ & Yes & None & $++\mathrm{Ni}$ & $++\mathrm{Ni},+\mathrm{Cr}$ \\
\hline 4 & $F$ & None & None & Yes & $\mathrm{Ni}$ & Yes & None & $++\mathrm{Ni}$ & $+\mathrm{Ni}$ \\
\hline 5 & $F$ & Yes & None & None & $\mathrm{Ni}$ & Yes & None & $++\mathrm{Ni}$ & $+\mathrm{Ni}$ \\
\hline 6 & $F$ & Yes & None & None & $\mathrm{Ni}$ & Yes & None & $++\mathrm{Ni}$ & $++\mathrm{Ni}$ \\
\hline 7 & $F$ & Yes & None & Yes & $\mathrm{Ni}$ & Yes & Yes & Neg & $+\mathrm{Ni}$ \\
\hline 8 & $F$ & Yes & None & None & $\mathrm{Ni}$ & Yes & Yes & Neg & $+\mathrm{Ni}$ \\
\hline 9 & M & Yes & None & Yes & $\mathrm{Ni}$ & None & Yes & Neg & $+\mathrm{Ni},+\mathrm{Cr}_{1}+\mathrm{Co},+\mathrm{Ti}$ \\
\hline 10 & $F$ & None & None & None & None & Yes & None & Neg & $+\mathrm{Ni},+\mathrm{Cr}$ \\
\hline 11 & M & None & None & None & None & None & None & Neg & $+\mathrm{Ni}$ \\
\hline \multicolumn{10}{|l|}{ Group 2B } \\
\hline 12 & $F$ & Yes & Yes & None & None & Yes & Yes & Neg & Neg \\
\hline 13 & $\mathrm{~F}$ & Yes & None & None & None & None & None & Neg & Neg \\
\hline 14 & $F$ & Yes & None & None & None & None & None & Neg & Neg \\
\hline 15 & M & Yes & None & None & None & None & None & Neg & Neg \\
\hline 16 & $F$ & Yes & None & None & None & Yes & Yes & Neg & Neg \\
\hline 17 & $F$ & Yes & None & None & None & Yes & None & Neg & Neg \\
\hline 18 & $F$ & None & None & None & None & None & None & Neg & Neg \\
\hline 19 & $F$ & Yes & None & Yes & None & Yes & None & $+\mathrm{Cr}$ & Neg \\
\hline 20 & M & Yes & None & None & None & None & None & $+\mathrm{Ni},+\mathrm{Co}$ & Neg \\
\hline 21 & $F$ & None & None & None & None & None & None & $+/-\mathrm{Co}$ & Neg \\
\hline 22 & M & Yes & Yes & None & None & None & None & $+/-\mathrm{Cr}$ & Neg \\
\hline
\end{tabular}

Ti did not activate cytokine production in any of the analysed cultures.

Cells from allergic patients with no prosthesis revealed a high reactivity to $\mathrm{Ni}$ and a mild reactivity to Mo, Co and $\mathrm{Cr}$, resulting in the prevalent induction of cytokines which were indicative of T-cell activation (IL-5, IL-2, IFN $\gamma$ ) and cytokines associated with monocyte/macrophage activation (IL-10).

Cells from allergic patients with painful prostheses revealed a high reactivity to $\mathrm{Cr}$ with statistically significant production of IL-7 and IL-12. Increased production of IL-5, RANTES, IL-7 and IL-17 was observed under
Ni stimulation. Comparison between patients with painful prostheses and allergic patients showed statistically significant differences $(p=0,027)$ only for IL-17. Co induced a statistically significant production of RANTES.

\section{Phase-contrast and laser scan confocal microscopy}

Cell interaction with metal particles was visualised by confocal microscopy, to evaluate cytoskeletal rearrangement and metal endocytosis.

Metal debris within cell cytoplasm can be observed as non-fluorescent dark areas of different shapes and sizes, which are not detectable in unstimulated cultures. 
Table 2 Number and percentage of stimulatory responses revealed by PT and LTT

\begin{tabular}{|c|c|c|c|c|c|}
\hline \multicolumn{6}{|c|}{ Group 1} \\
\hline & Number of cases & $\%$ & & Number of cases & $\%$ \\
\hline PT+ & $7 / 8$ & 87,5 & $\mathrm{LTT}+$ & $6 / 8$ & 75 \\
\hline PT- & - - & & LTT- & $2 / 8$ & 25 \\
\hline PT+/- & $1 / 8$ & 12,5 & & & \\
\hline \multicolumn{6}{|c|}{ Group 2A } \\
\hline & Number of cases & $\%$ & & Number of cases & $\%$ \\
\hline PT+ & $6 / 11$ & 54,5 & $\mathrm{LTT}+$ & $11 / 11$ & 100 \\
\hline PT- & $5 / 11$ & 45,5 & LTT- & - & - \\
\hline \multicolumn{6}{|c|}{ Group 2B } \\
\hline & Number of cases & $\%$ & & Number of cases & $\%$ \\
\hline PT+ & $2 / 11$ & 18 & $\mathrm{LTT}+$ & - & - \\
\hline PT- & $7 / 11$ & 64 & LTT- & $11 / 11$ & 100 \\
\hline $\mathrm{PT}+/-$ & $2 / 11$ & 18 & & & \\
\hline
\end{tabular}

Generally, Ti, Mo and Co seemed to have non-toxic effects resulting in well-preserved cell morphology, while marked cytoskeletal alterations were found following exposure to $\mathrm{Ni}$ and $\mathrm{Cr}$. Representative images of cells stimulated by different metals $(0.1 \mathrm{mM})$ are shown in Fig. 3 . Panels A and B represent phase contrast $(\mathrm{A} 1 \rightarrow \mathrm{A} 8)$ and fluorescent images $(\mathrm{B} 1 \rightarrow \mathrm{B} 8)$ with overlapping counterstained nuclei.

No signs of cellular distress were found in either untreated cells (A1, B1) and Ti-stimulated cells (A2, B2). Some minor cytoskeletal modifications were seen in $\mathrm{Co}$ (A3, B3) and Mo (A4, B4) -treated cells, which in some cases were similar to controls. Conversely, destruction of cytoskeletal components was evident in $\mathrm{NiCl}_{2}$ (A5, B5) and $\mathrm{Ni}$ (A6, B6) -treated samples. Moreover, $\mathrm{CrCl}_{3}$ (A7, B7) and $\mathrm{Cr}(\mathrm{A} 8, \mathrm{~B} 8)$ caused multiple defects in the cytoskeleton, and the cytoplasm appeared markedly damaged or partially destroyed.

Confocal optical sections (z-stacks) confirmed the internalisation of metal particles, which appeared in sequential images as multiple dark areas inside the cytoplasm (Fig. 4a). Similar results were obtained in all samples. Representative images are shown in Fig. 4b-f.

Untreated cells (Fig. 4b) showed round or oval shapes, with central, slightly enlarged nuclei. No dark areas were observed inside the cytoplasm.

Ti, Mo and Co-stimulated cells appeared similar to controls (data not shown).

In the attempt to obtain a quantitative evaluation, the percentages of cells containing metal debris were calculated.

Generally, high percentages of damaged cells were found in $\mathrm{Ni}$ and $\mathrm{Cr}$-stimulated cells, while low percentages of positive cells were found in $\mathrm{Ti}$, Mo and Co- stimulated cells, except for the few patients with specific $\mathrm{Ti}$, Mo or Co-sensitivity.

Similar behavior was observed for the two concentrations of sensitizer metals, but statistical significance was not reached, owing to the lack of uniformity in cell counts among the patients. In fact, at the concentration of $0.1 \mathrm{mM}$, the percentage of cells containing even a little amount of metals, varies from 2 to $17 \%$ for $\mathrm{Ti}$, from 2 to $18 \%$ for Mo, and from 4 to $22 \%$ for Co.

On the other hand, $\mathrm{NiCl}_{2}$ and $\mathrm{Ni}$-treated cells (Fig. 4c,d) showed clusters of metal debris surrounding the nucleus, which was still completely enclosed in the cytoplasm, as well as internalised aggregated particles in fragmented cells. The percentage of damaged cells varied from 70 to $82 \%$ for $\mathrm{NiCl}_{2}$ and from 47 to $55 \%$ for $\mathrm{Ni}$. Multiple dark holes due to phagocytosed particles were also found in $\mathrm{CrCl}_{3}$ and $\mathrm{Cr}$-treated cells (Fig. 4e,f), with percentage of damaged cells varied from 40 to $48 \%$ and from 40 to $44 \%$, respectively.

Morphological changes, such as disruption of cell membrane and disappearance of nuclei, indicated severe cell injury.

\section{Clinical outcome}

All Group 1 patients with hypersensitivity diagnosed by PT and LTT underwent TKA with a non-allergic implant (Genesis II, Smith \& Nephew, Memphis, TN), characterised by an Oxidised Zirconium femoral component, and all PolyEthylene (PE) tibial and patellar components.

At the 12-month post-surgical evaluation, no complications were observed, and all patients referred a good outcome.

On the basis of clinical evidence, seven of the Group 2A patients with hypersensitivity, diagnosed by LTT alone or PT plus LTT, underwent revision arthroplasty using a Nickel-free implant with an Oxidized Zirconium femoral component (Legion OxZr/PE/Ti, Smith \& Nephew, Warsaw, IN) or a Zirconium Nitride multicoating implant (E-motion multicoating NiZr/PE, BBraun, Melsungen AG). The other four patients were monitored by a strict followup and treated by pain-controlling drugs, owing to the individual risk of possible complications due to concomitant disease. They underwent revision surgery using the Nickelfree implant (Legion $\mathrm{OxZr} / \mathrm{PE} / \mathrm{Ti}$, Smith \& Nephew, Warsaw, IN). At the 12-month postoperative follow-up, no complications were observed, and patients referred relief from symptoms and a satisfactory functional recovery. Two subjects referred clinical improvement, but with persistent swelling and pain. They are still under observation in order to understand the reason for this partial recovery.

Group 2B patients, who all showed negative LTT but different responses to PT, were considered as not allergic to metals. In three out of eleven patients who had undergone a second surgical procedure with a Nickel- 

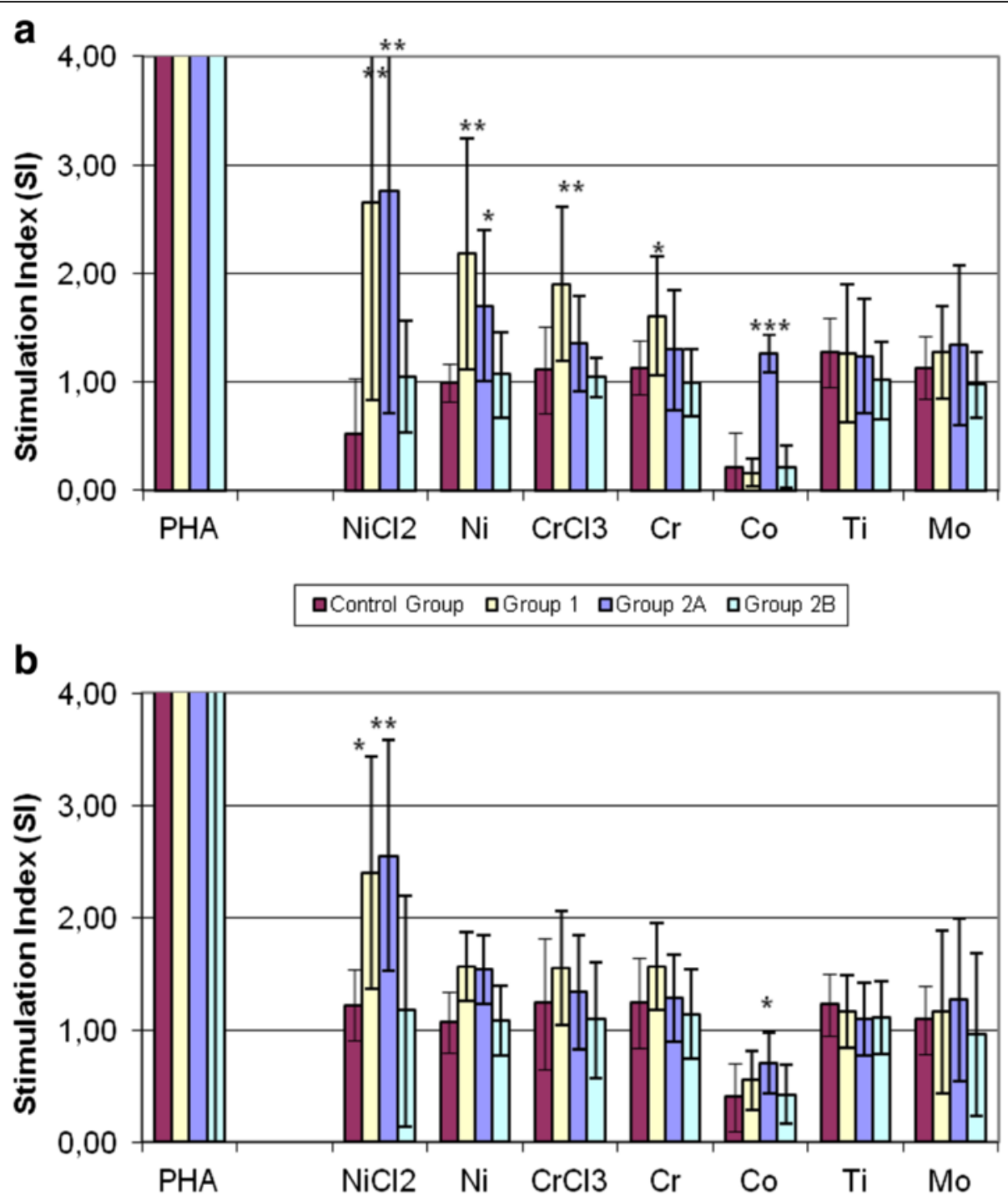

aControl Group aGroup 1 aGroup 2A aGroup 2B

Fig. 1 a, $\mathbf{b}$ Lymphocyte Transformation Test Response. The effect of various metals and PHA on the proliferation rate of patients' and controls' lymphocytes: mean lymphocyte response (SI) of each of the three patient groups compared to controls. Group $1=$ patients scheduled for pTKA, labelling for a clinical history of metal allergy; Group 2A =TKA patients with pain and clinical signs of metal allergies; Group 2B=TKA patients with pain and no clinical signs of metal allergies; Group $3=$ Control subjects. Metal concentrations: $(\mathbf{a})=0.1 \mathrm{mM} ;(\mathbf{b})=0,01 \mathrm{mM}$. Asterisks indicate: $\left(^{*}\right) p<0.05 ;(* *) p<0.005 ;\left({ }^{* *}\right) p<0.0001$

free implant (Legion OxZr/PE/Ti, Smith \& Nephew, Warsaw, IN), the pain had disappeared. The other patients, kept under clinical observation and treated with oral analgesics and periodical steroid drug administration, referred persistent symptoms and a variable joint function.

\section{Discussion}

Several studies were performed to analyse the possible relationship between metal hypersensitivity and painful or poorly-functioning prostheses. The individual conditions which may favour the onset of post-surgical complications are as yet unknown. None of the clinical or laboratory analyses prove that these complications may be caused by metal particles released from implants or by pre-existing metal sensitivity. Nevertheless, in vitro testing for metal allergies is recommended for patients undergoing arthroplasty who have known hypersensitivity reactions.

In our study, all laboratory results were considered in the decision-making process, and treatment options were tailored according to the patient's needs. Even so, some patients with painful prostheses referred only a 

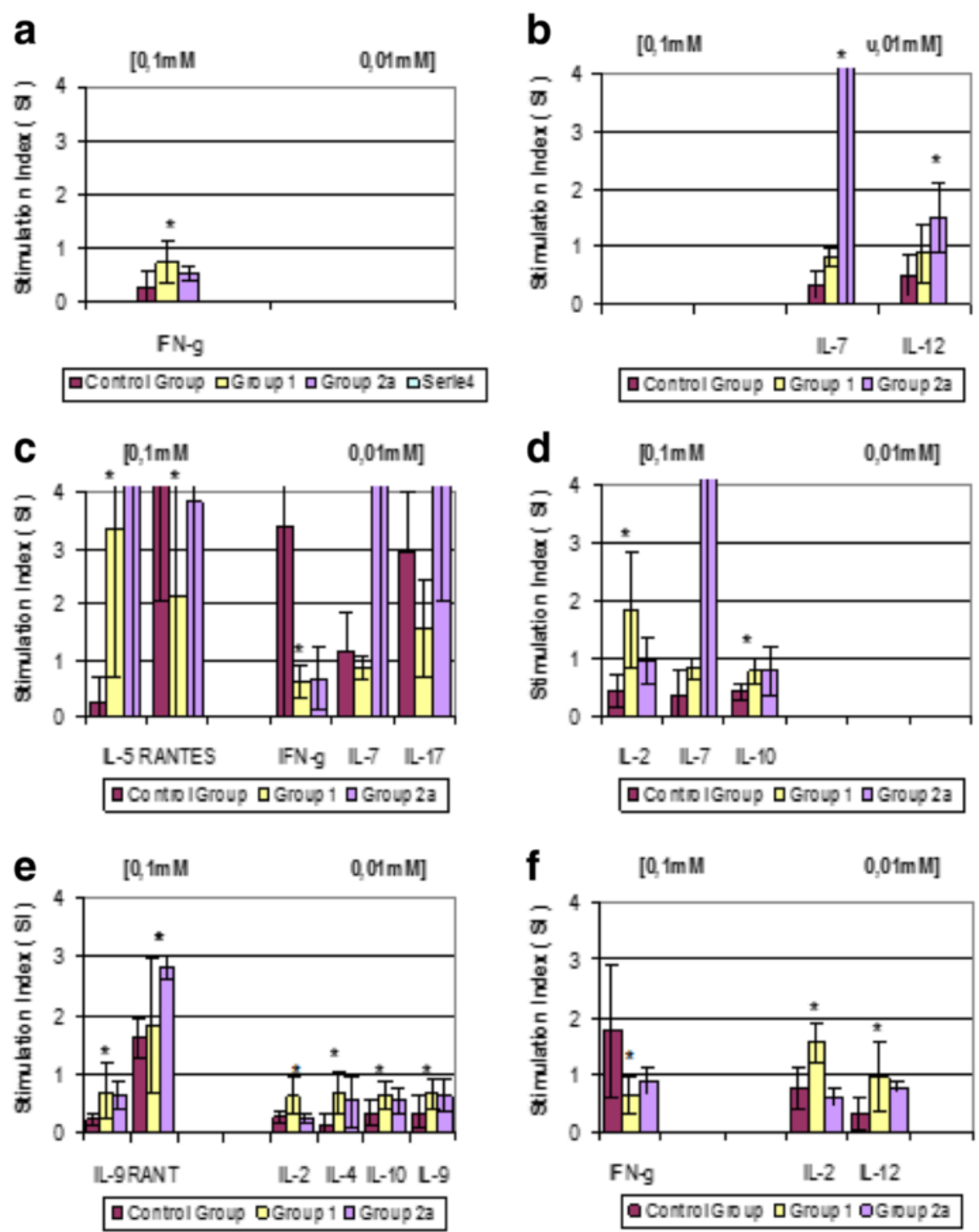

Fig. 2 a-f Cytokine production in response to metals. Cytokine production in response to metals ( $0.1 \mathrm{mM}$ and $0.01 \mathrm{mM})$ in the two patient groups compared to controls. Group 1 = patients scheduled for PTKA, labelling for a clinical history of metal allergy; Group 2A = TKA patients with pain and clinical signs of metal allergies; Group 2B $=$ TKA patients with pain and no clinical signs of metal allergies; Group $3=$ Control subjects. Metals: $(\mathbf{a})=$ Chromium chloride; $(\mathbf{b})=$ Chromium; $(\mathbf{c})=$ Nickel chloride; $(\mathbf{d})=$ Nickel; $(\mathbf{e})=$ Cobalt; $(\mathbf{f})=$ Molybdenum. $(\mathrm{SI}=$ mean of Stimulation Index). Asterisks indicate: $\left(^{*}\right) p<0.05 ;\left(^{* *}\right) p<0.005$

partial recovery after revision surgery. As discussed elsewhere, the persistence of post-surgical pain is not clearly defined [28, 29], but Macrae reports that surgery itself is the second most common cause of this problem [30]. A high failure rate in a revision series (about $80 \%$ ) has been reported in cases of undefined pain origin [31]. The persistence of pain may be a consequence of underlying conditions, or of surgical complications. Wylde et al. assess that $44 \%$ of patients undergoing TKA suffer from persistent post-surgical pain of varying severity
[32] and revision surgery may also lead to uncertain clinical results.

These data highlight the need to have as much information as possible concerning the nature of pain. Moreover, there is an urgent need to develop in vitro tests to achieve better understanding of this problem. Current laboratory methods, used individually, failed to provide a sure diagnosis. To offset the limitations of each method, a combined approach evaluating both PT and LTT was used to detect systemic metal reactivity. When a diagnostic test is 

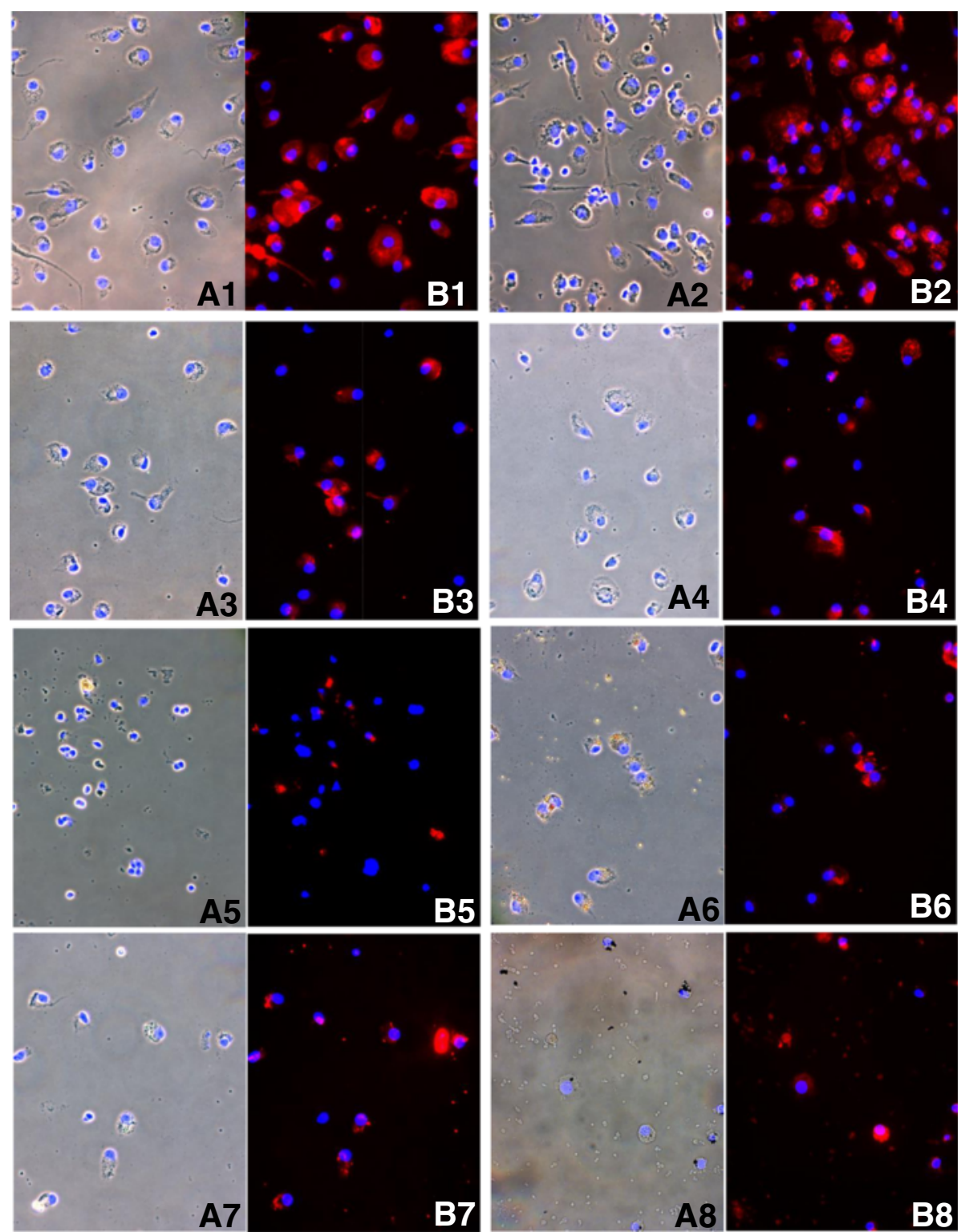

Fig. 3 Phase-contrast microscopy. Representative images of cells stimulated by different metals $(0.1 \mathrm{mM})$. Panels $\mathbf{a}$ and $\mathbf{b}$ represent phase contrast $(A 1 \rightarrow A 8)$ and fluorescent images $(B 1 \rightarrow B 8)$, overlapped with counterstained nuclei. (A1-B1: untreated cells; A2-B2: Ti; A3-B3: Co; A4-B4: Mo; A5-B5: NiCl $;$; A6-B6: Ni; A7-B7: $\mathrm{CrCl}_{3} ; A 8-B 8$ : Cr-treated samples. (Phalloidin TRITC-conjugate and Hoechst 33258). (1-3; 5-7 = 40X original magnification), $(4 ; 8=63 \times$ original magnification)

unable to specifically identify a clinical condition, it must be double-checked with another test to confirm a preliminary diagnosis or clarify contradictory responses.

PT may not reflect the immunological response at the implant site. A positive PT indicates an allergen-specific cutaneous reaction, but does not clearly signify the development of metal sensitivity. PT itself may also induce sensitization. Moreover, strong reactions indicate true positivity, but it is very difficult to interpret a doubtful result [33]. Nevertheless, it must always be considered within the overall clinical context, or be supported by other findings. Only more in-depth analysis can rule out or confirm allergic response and identify the specific sensitiser metal.

LTT may be more useful than PT in allergic patients, ruling out a direct contact with allergens as well as doubtful PT results. Therefore, considering that PT alone is inadequate for formulating a diagnosis or for deciding on revision surgery, we explored the potential of LTT as a confirmatory test. The limited agreement between PT and LTT shown by the Kappa coefficient is probably due to the small cohorts analysed. 
a

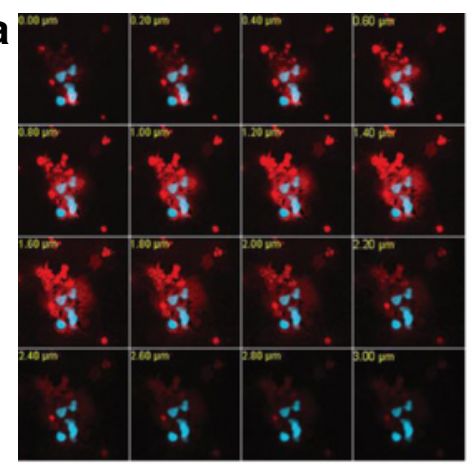

c

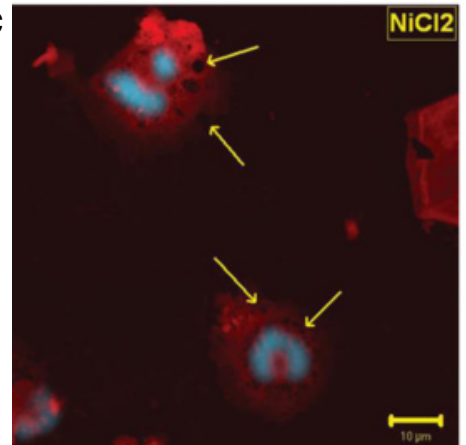

e

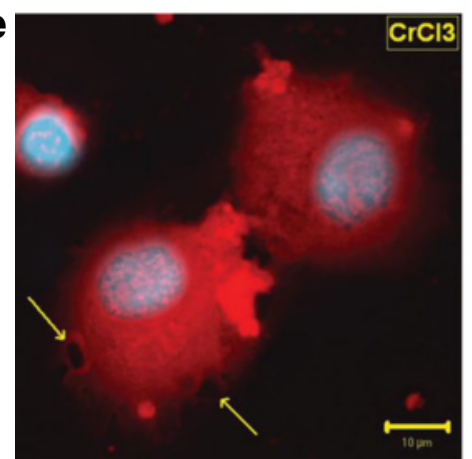

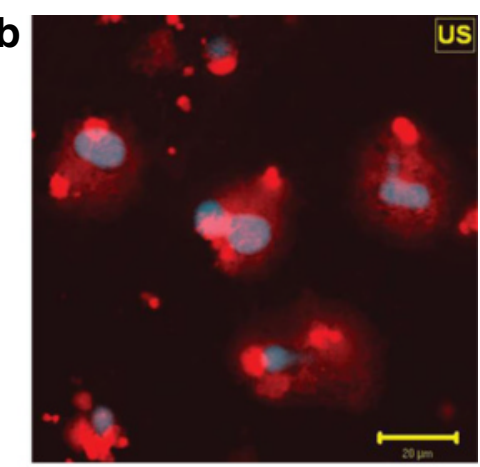

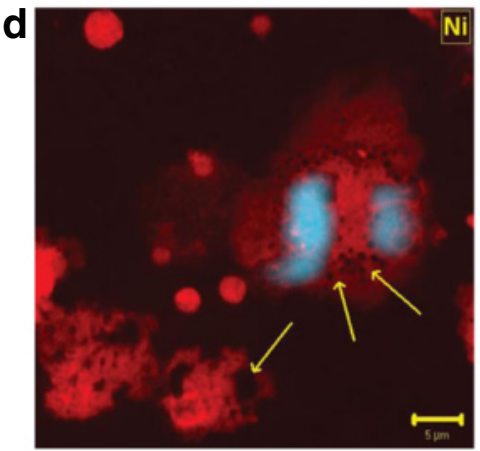

f

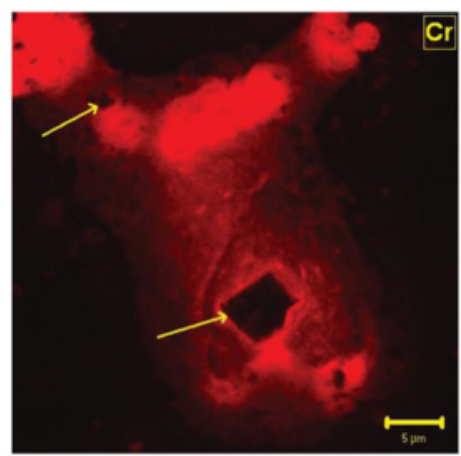

Fig. 4 a-f Confocal Microscopy. Representative images of z-stacks optical sections (a), untreated cells (b), $\mathrm{NiCl}_{2}(\mathbf{c}), \mathrm{Ni}(\mathbf{d}), \mathrm{CrCl}_{3}(\mathbf{e})$, and $\mathrm{Cr}(\mathbf{f})$-stimulated cells. (Phalloidin TRITC-conjugate, Hoechst 33258). (Phalloidin TRITC-conjugate, Hoechst 33258). b=40x, c,e = 63x, d,f = 100x original magnification

Sometimes PT reactivity was not confirmed by LTT. False-positive or negative reactions in patients affected with contact dermatitis have been documented by Brasch et al. and Sarma [34, 35]. In our experimental conditions, $87 \%$ of Group 1 patients tested positive to PT. LTT confirmed and sometimes expanded the diagnosis of metal reactivity in $75 \%$ of cases. Negative LTT results were explained as skin-specific sensitivity.

All Group 2A patients were found positive to LTT but only $55 \%$ to PT. This may be indicative of a systemic sensitisation, but it means that PT failed to identify sensitised subjects in $45 \%$ of cases. At the same time, all Group 2B patients were found negative to LTT. Again, a clearly negative response to PT was detected in only $64 \%$ of cases. Since comparison with other methods is lacking, this constitutes a serious limit to the clinical decision process.
These results supported our working hypothesis, whereby LTT is a more suitable method for testing systemic allergies and differentiating dermal from implant-induced hypersensitivity reactions. However, the lack of overlap suggests that these tests are complementary but not equivalent. In fact, they recognise different biological mechanisms which are only partially correlated. This is borne out by the comparison of mean LTT SI values among the 4 groups. In fact, allergic subjects affected by knee arthritis and hypersensitivity to metals diagnosed by PT and LTT had comparable SI values to those of patients with painful arthroplasty, while patients with painful arthroplasty but no clinical signs of sensitisation showed similar results to controls. No significant differences were detected between the two concentrations of sensitiser metals. 
LTT response to $\mathrm{NiCl}_{2}$ and $\mathrm{Ni}$ was significantly higher in Group 1 and Group 2A patients. Statistically significant effects were also determined by $\mathrm{CrCl}_{3}$ and $\mathrm{Cr}$ in Group 1 patients, despite the fact that all values constantly remained below the SI threshold value.

Interestingly, Co produced a remarkable degree of proliferative response only in Group 2A patients. The high statistical significance of these findings is consistent with the clinical characteristics of these patients. Moreover, a dose-response relation between the two metal concentrations was also observed. No statistically significant differences in $\mathrm{Ti}$ and Mo responses were detected among the four groups.

These findings showed that positive LTT response may indicate patients who have a predisposition to develop pathological reactions to implanted devices, and confirm the suspicion of metal allergy in patients with painful prostheses. Thus in case of limited resources, we suggest that LTT should be preferred to PT.

Cytokine assay may be reserved for patients undergoing revision surgery.

Regarding cytokine production, in allergic patients with no prosthesis Ni caused a statistically significant increase in IL-5, which is considered an allergen-specific cytokine. Czarnobilska et al. showed that Nickel-induced IL-5 production is related to the intensity of PT response and might be a useful marker to distinguish between allergic and non-allergic subjects [36].

IL-5 was also reported to be secreted in vitro by nickel-stimulated peripheral blood mononuclear cells in $\mathrm{Ni}$-allergic patients. All these results indicate that type 2 response might contribute to the immunopathogenesis of contact hypersensitivity [37-39].

Also a statistically significant increase in IL-2 was evidenced, in agreement with previous reports that indicate IL-2 as an early occurring type-1cytokine in patients with $\mathrm{Ni}$ allergic contact dermatitis [40].

A statistically significant production of RANTES, which may recruit leukocytes at the site of inflammation, was found. Other cytokines potentially involved in allergic responses (IL-4, IL-9) were detected. IL-7 regulates Th1/Th2 cytokine production, while IL-2 and IL-10 can play immune-suppressive or stimulatory roles. An increase in IL-2 and a decrease in IFN- $\gamma$ may induce a switch of the $\mathrm{T}$ helper lymphocytes. The up-regulation of Th2 and Th9 cells and the down-regulation of Th1 cells reveal a typical allergic pattern in this group of patients. All these data are coherent with the significantly higher proliferative response to $\mathrm{Ni}$, found by the LTT test, in this group.

In allergic patients with painful prostheses the high reactivity to Co enhanced the production of RANTES, which may recruit leukocytes at the inflammation site. Other metals induced the production of cytokines associated with monocyte-macrophage activation, which plays a role in the regulation of type1/type2 cytokine balance (IL-12). Elevated IL-12 levels have been reported by Inomoto et al. in the pseudosynovial fluid of patients with aseptic loosening of hip prostheses [41].

A statistically significant increase was observed in IL-7 production under $\mathrm{Cr}$ stimulation and IL-17 production under Ni stimulation. These osteogenic cytokines can stimulate RANKL production. As is known, the RANKRANKL system stimulates osteoclast activation, increasing bone resorption [42, 43]. Increased levels of these cytokines are consistent with the clinical characteristics of the patients analysed, and can be related to implant failure. Also in this group of patients the increased response to $\mathrm{Ni}$ and Co observed by LTT are coherent with the production of osteoclastogenic cytokines which are specific for these patients.

This is in accordance with Summer B. et al. that showed that patients with complicated total joint arthroplasty and concomitant $\mathrm{Ni}$ patch test reactivity had a predominant IL-17 response. This suggests that a potentially increased risk of complications following prosthesis implantation might be based on the evaluation of IL-17 [4].

Regarding the cytokine profile, the observed data are insufficient to support the need for routine cytokine assay as a preliminary test. In our experimental conditions, a different response was found in the two patient groups. All metals induced a mixed Th1 and Th2-type cytokine production. Despite the small number of cases, the prevalence of a Th2-cytokine pattern may be used to identify predisposition to the development of allergic diseases. At the same time, the selective presence of osteoclastogenic cytokines as IL-17 and IL-7 and the evidence of RANTES and IL-12, may be used as predictors of a negative outcome in patients with painful prosthesis.

However, because of the high inter-individual and intra-individual variability of cytokine expression, a population study needs to be performed to ascribe prognostic values to specific cytokines.

An alternative approach is represented by molecular analysis of cytokine pattern together with histological evaluation of the periimplant tissue, in patients undergoing revision surgery.

Hercus B. et al. analyzed mRNA expression of TH1 (IFN- $\gamma$, TNF- $\beta$, IL-2, IL-12) and TH2 (IL-4, IL-5, IL-6, IL-10) cytokines present at the bone-implant interface of aseptically loosened joints, and demonstrated a predominance of TH1 over TH2 response [44].

Recently, Thomas suggested a combined approach to evaluate a possible link between a specific cytokine expression pattern and periimplant tissue analysis, in patients with TKA failure. An high expression of IFN- $\gamma$ and IL-2 was evidenced with semiquantitative real-time RT-PCR $[26,45]$. 
Regarding histological analysis of periprosthetic tissues, past investigations evidenced the presence of metallic aggregates in the perilesional area, as well as perivascular infiltrations of T-cells and macrophages in the tissue sections $[46,47]$.

Davies A.P. et al. described arthroplasty failure in association with the histological evidence of periprosthetic lymphocytic infiltration and macrophage containing metal debris [48].

Witzleb W.C. et al. reported that the presence of diffuse and perivascular lymphocytic infiltration in periprosthetic tissue may be considered to be a characteristic histological pattern of tissue reactions to metal particles [49].

Other Authors analysed some histological parameters and cytokine levels in tissue and synovial fluids from patients undergoing primary total hip/knee replacement and from patients requiring revision for aseptic loosening. Statistically significant increases in cytokine levels and in macrophage infiltrate were found in samples from patients with aseptic loosening, compared to patients undergoing primary surgery $[50,51]$.

For histopathological classification of the hypersensitivity reactions in periprosthetic tissue, a standardized consensus classification was established [52].

Nevertheless, microscopy approach remains a qualitative method, generally referred to limited groups of cells. Other limitations have arisen from the heterogeneity of implant devices, source and location of tissue samples, patient characteristics and because of the absence of proper control groups.

All of these investigations concern patients requiring hip or knee revision surgery for ascertain aseptic loosening of prosthesis.

Our study design aimed to identify common laboratory methods for the preventive evaluation of potentially allergic patients and for the early diagnosis of aseptic loosening in patients whit painful prosthesis.

Phase-contrast microscopy was preliminarily used on PBMC to reveal changes in cytoskeletal structure, and Laser Scan Confocal analysis was applied to visualise solid particles internalisation and their effects on cytoskeleton rearrangement.

Generally speaking, phase-contrast microscopic evaluation revealed that $\mathrm{Ti}, \mathrm{Mo}$ and Co had non-toxic effects resulting in well-preserved cell morphology.

Conversely, after exposure to $\mathrm{Ni}$ and $\mathrm{Cr}$, the number of cells appeared to be strongly reduced and marked cytoskeletal alterations were revealed.

Also LSCM observation confirmed the relatively low toxicity of $\mathrm{Ti}$, Mo and $\mathrm{Co}$, while under $\mathrm{Ni}$ and $\mathrm{Cr}$ stimulation, an high percentage of cells with a considerable amount of metals inside was evidenced. Enlarged cells with multiple defects in cytoskeletal organisation, caused by individual and aggregated internalised particles in perinuclear areas, confirmed the cytotoxic effect of these metals.

Phase-contrast and Laser Scan Confocal microscope observations of PBMC did not provide a statistically significant quantitative evaluation. However, no diagnostic response, but only the proof of metal particles internalization, was expected.

\section{Conclusions}

In conclusion, to achieve an improvement in clinical practice, a PT confirmed by LTT could be introduced as standard procedure. This would allow the identification of subjects who are likely to develop implant-related hypersensitivity reactions. At the same time, it would avoid the development of allergies from joint implantation, and reveal any reactions due to implant compounds.

\section{Abbreviations}

Co: Cobalt powder; Cr: Chromium powder; $\mathrm{CrCl}_{3}$ : Chromium (III) chloride; CRP: C-reactive protein; ESR: Erythrocyte sedimentation rate; KSS: Knee society score; LSCM: Laser scan confocal microscopy; LTT: Lymphocyte transformation test; MHC II: Class II major histocompatibility complex; Mo: Molybdenum nanopowder; Ni: Nickel nanopowder; $\mathrm{NiCl}_{2}$ : Nickel (II) chloride; PBMC: Peripheral blood mononuclear cells; PE: PolyEthylene; PHA: Phytohemagglutinin; PT: Patch test; PTKA: Primary total knee arthroplasty; ROM: Range of motion; SI: Stimulation index; Ti: Titanium powder; TKA: Total knee arthroplasty; WOMAC: Western Ontario and McMaster Universities Osteoarthritis Index

\section{Acknowledgments}

We would like to thank Dr. Marie Pierre Piccinni and Dr. Rosanna Matucci for their helpful contribution and their expert support in technical procedures.

\section{Funding}

This work is supported by an unrestricted grant from Smith \& Nephew and by F.I.R.M.O. Fondazione Raffaella Becagli (to Maria Luisa Brandi).

\section{Availability of data and materials}

All relevant information are included within the article. Please address requests for additional data and materials to the corresponding author

\section{Authors' contributions}

AMC designed and performed experiments, analysed the data and wrote the paper, SC collaborated to perform cytokine assays, RC set up cell culture experiments and performed Lymphocyte Transformation Tests, GDZT performed immunofluorescence-staining for phase-contrast and confocal microscopy, RZ collaborated to perform Confocal Microscopy, Ml and CC were responsible for screening and selection of patients and for all clinical interventions, MLB provided critical revision for intellectual content and final approval of the manuscript All authors read and approved the final paper.

Authors' information

Not applicable.

\section{Competing interests}

The authors declare that they have no competing interests.

\section{Consent for publication}

All participants gave informed consent for publication of the data.

\section{Ethics approval and consent to participate}

Ethical approval for this study has been obtained from the "Comitato Etico per la Sperimentazione Clinica dei Medicinali" Azienda Ospedaliera Universitaria Careggi (Prot. n. 2013/00 Rif. n. 68/13).

Informed consent was obtained from all participants for research studies and presented data. 


\section{Received: 27 April 2016 Accepted: 14 November 2016} Published online: 23 November 2016

\section{References}

1. Ritter MA. The anatomical graduated component total knee replacement. A long-term evaluation with 20-years survival analysis. J Bone Joint Surg Br. 2009:91(6):745-9.

2. Carulli C, Matassi F, Nistri L, Civinini R, Innocenti M. Long-term survival of a flat-on-flat total condylar knee arthroplasty fixed with a hybrid cementing technique for tibial components. J Long Term Eff Med Implants. 2012;22(4):305-12.

3. Kurtz SM, Lau E, Ong K, Zhao K, Kelly M, Bozic KJ. Future young patient demand for primary and revision joint replacement: national projections from 2010 to 2030. Clin Orthop Relat Res. 2009;467(10):2606-12.

4. Summer B, Paul C, Mazoochian F, Rau C, Thomsen M, Banke I, et al. Nickel (Ni) allergic patients with complications to $\mathrm{Ni}$ containing joint replacement show preferential IL-17 type reactivity to $\mathrm{Ni}$. Contact Dermatitis. 2010:63(1):15-22.

5. Villano M, Carulli C, Puccini S, Soderi S, Innocenti M. Painful knee prosthesis: surgical approach. Clin Cases Miner Bone Metab. 2011;8(2):26-8.

6. Thomas P, Braathen LR, Dörig M, Auböck J, Nestle F, Werfel T, et al. Increased metal allergy in patients with failed metal-on-metal hip arthroplasty and periimplant T lymphocytic inflammation. Allergy. 2009; 64(8):1157-65.

7. Hallab N, Merritt K, Jacobs JJ. Metal sensitivity in patients with orthopaedic implants. J Bone Joint Surg Am. 2001:83:428-36.

8. Jacobs JJ, Hallab NJ, Skipor AK, Urban RM. Metal degradation products: a cause for concern in metal-metal bearings? Clin Orthop Relat Res. 2003; 417:139-47.

9. Basko-Plluska JL, Thyssen JP, Schalock PC. Cutaneous and systemic hypersensitivity reactions to metallic implants. Dermatitis. 2011;22:65-79.

10. Michel R, Nolte M, Reich M, Loer F. Systemic effect of implanted prostheses made of cobalt-chromium alloys. Arcgh Orthop Trauma Surg. 1991;110:61-74.

11. Urban RM, Jacobs JJ, Tomlinson MJ, Gavrilovic J, Black J, Peoc'h M. Dissemination of wear particles to the liver, spleen, and abdominal lymph nodes of patients with hip or knee replacement. J Bone Joint Surg Am. 2000;82(4):457-76.

12. Hallab NJ, Caicedo M, Epstein R, McAllister K, Jacobs JJ. In vitro reactivity to implant metals demonstrates a person dependant association with both T-cell and B-cell activation. J Biomed Mat Res. 2010;92(2):667-82.

13. Niki Y, Matsumoto $H$, Otani $T$, Yatabe $T$, Kondo M, Yoshimine $F$, et al. Screening for symptomatic metal sensitivity: a prospective study of 92 patients undergoing total knee arthroplasty. Biomaterials. 2005;26:1019-26

14. Abu-Amer Y, Darwech I, Clohisy JC. Aseptic loosening of total joint replacements: mechanisms underlying osteolysis and potential therapies. Arthritis Res Ther. 2007;9(Suppl1):S6

15. Gawkrodger DJ. Metal sensitivities and orthopaedic implants revisited: the potential for metal allergy with the new metal-on-metal joint prostheses. Br J Dermatol. 2003;148(6):1089-93.

16. Looney RJ, Schwarz EM, Boyd A, O'Keefe RJ. Periprosthetic osteolysis: an immunologist's update. Curr Opin Rheumatol. 2006;18(1):80-7.

17. Belsito DV. The diagnostic evaluation, treatment, and prevention of allergic contact dermatitis in the new millennium. J Allergy Clin Immunol. 2000;105:409-20.

18. Mowad CM. Patch testing: pitfalls and performance. Curr Opin Allergy Clin Immunol. 2006;6:340-4.

19. Hallab NJ. Lymphocyte transformation testing for quantifying metal implant related hypersensitivity responses. Dermatitis. 2004;15:82-90.

20. Thyssen JP, Menné T, Schalock PC, Taylor JS, Maibach HI. Pragmatic approach to the clinical workup of patients with putative allergic disease to metallic orthopaedic implants before and after surgery. Br J Dermatol. 2011;164(3):473-8.

21. Innocenti M, Civinini R, Carulli C, Matassi F, Villano M. The 5-year results of an oxidized zirconium femoral component for TKA. Clin Orthop Relat Res. 2010;46(5):1258-63.

22. lida T, Minoda $Y$, Kadoya $Y$, Matsui $Y$, Kobayashi A, Iwaki H, et al. Mid-term clinical results of alumina medial pivot total knee arthroplasty. Knee Surg Sports Traumatol Arthrosc. 2012;20(8):1514-9.

23. Wilkinson DS, Fregert S, Magnusson B, Bandmann HJ, Calnan CD,

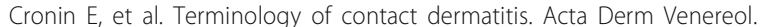
1970;50:287-92.
24. Bourke J, Coulson I, English J. British association of dermatologists. Guidelines for care of contact dermatitis. Br J Dermatol. 2001;145:877-85.

25. Bourke J, Coulson I, English J. British Association of Dermatologists Therapy Guidelines and audit subcommittee. Guidelines for the management of contact dermatitis: an update. Br J Dermatol. 2009;160(5):946-54.

26. Thomas P, von der Helm C, Schopf C, Mazoochian F, Frommelt L, Gollwitzer $H$, et al. Patients with intolerance reactions to total knee replacement: combined assessment of allergy diagnostics, periprosthetic histology, and peri-implant cytokine expression pattern. Biomed Res Int. 2015;2015:910156.

27. Altman DG. Practical statistics for medical research. 1st ed. London: Chapman \& Hall; 1991.

28. Piscitelli P, Iolascon G, Innocenti M, Civinini R, Rubinacci A, Muratore M, et al. Painful prosthesis: approaching the patient with persistent pain following total hip and knee arthroplasty. Clin Cases Miner Bone Metab. 2013:10(2):97-110

29. Kehlet $H$, Jensen TS, Woolf CJ. Persistent postsurgical pain: risk factors and prevention. Lancet. 2006;367:1618-25.

30. Macrae WA. Chronic postsurgical pain: 10 years on. Br J Anaesth. 2008;101:77-86.

31. Laskin RS. The painful knee. Orthopedics. 1999;22(9):869-70.

32. Wylde V, Hewlett S, Learmonth ID, Dieppe P. Persistent pain after joint replacement: prevalence, sensory qualities, and post-operative determinants. Pain. 2011;152:566-72.

33. Nosbaum A, Vocanson M, Rozieres A, Hennino A, Nicolas JF. Allergic and irritant contact dermatitis. Eur J Dermatol. 2009;19(4):325-32.

34. Brasch J, Henseler T, Aberer W, Bäuerle G, Frosch PJ, Fuchs T, et al. Reproducibility of patch tests. A multicenter study of synchronous left versus right sided patch tests by the German contact dermatitis research group. J Am Acad Dermatol. 1994;3:584-91.

35. Sarma N. Late reaction, persistent reaction and doubtful allergic reaction: the problems of interpretation. Indian J Dermatol. 2009;54(1):56-8.

36. Czarnobilska E, Jenner B, Kaszuba-Zwoinska J, Kapusta M, Obtułowicz K, Thor $P$, et al. Contact allergy to nickel: patch test score correlates with IL5, but not with IFN gamma nickel specific secretion by peripheral blood lymphocytes. Ann Agric Environ Med. 2009;16(1):37-41.

37. Buchwald D, Lundeberg L. Impaired responses of peripheral blood mononuclear cells to nickel in patients with nickel-allergic contact dermatitis and concomitant atopic dermatitis. Br J Dermatol. 2004;50(3):484-92.

38. Borg L, Christensen JM, Kristiansen J, Nielsen NH, Menné T, Poulsen LK. Nickel-induced cytokine production from mononuclear cells in nickelsensitive individuals and controls. Cytokine profiles in nickel-sensitive individuals with nickel allergy-related hand eczema before and after nickel challenge. Arch Dermatol Res. 2000;292(6):285-91.

39. Jakobson E, Masjedi K, Ahlborg N, Lundeberg L, Karlberg AT, Scheynius A. Cytokine production in nickel-sensitized individuals analysed with enzymelinked immunospot assay: possible implication for diagnosis. Br J Dermatol. 2002;147(3):442-9.

40. Falsafi-Amin H, Lundeberg L, Bakhiet M, Nordlind K. Early DNA synthesis and cytokine expression in the nickel activation of peripheral blood mononuclear cells in nickel-allergic subjects. Int Arch Allergy Immunol. 2000;123:170-6.

41. Inomoto M, Miyakawa S, Mishima H, Ochiai N. Elevated interleukin12 in pseudosynovial fluid in patients with aseptic loosening of hip prosthesis. J Orthop Sci. 2000;5:369-73.

42. Lee SK, Surh CD. Role of interleukin 7 in bone and T cell homeostasis. Immunol Rev. 2005;208:169-80.

43. Toraldo G, Roggia C, Qian WP, Pacifici R, Weitzmann MN. IL7 induces bone loss in vivo by induction of receptor activator of nuclear factor kappa B ligand and tumor necrosis factor alpha from T cells. Proc Natl Acad Sci USA. 2003;100(1):125-30.

44. Hercus B, Saeed S, Revell PA. Expression profile of T cell associated molecules in the interfacial tissue of aseptically loosened prosthetic joints. J Mater Sci Mater Med. 2002;13(12):1153-6.

45. Thomas P, von der Helm C, Schopf C, Thomsen M, Frommelt L, Schneider J, et al. Peri-implant histology and cytokine pattern in metal-allergic knee arthroplasty patients with improvement after revision with hypoallergenic materials. Semin Arthroplast. 2012;23(4):268-72.

46. Gallo J, Vaculova J, Goodman SB, Konttinen YT, Thyssen JP. Contributions of human tissue analysis to understanding the mechanisms of loosening and osteolysis in total hip replacement. Acta Biomater. 2014;10(6):2354-66.

47. Ng VY, Lombardi Jr AV, Berend KR, Skeels MD, Adams JB. Perivascular lymphocytic infiltration is not limited to metal-on-metal bearings. Clin Orthop Relat Res. 2011;469(2):523-9. 
48. Davies AP, Willert HG, Campbell PA, Learmonth ID, Case CP. An unusual lymphocytic perivascular infiltration in tissues around contemporary metal-on-metal joint replacements. J Bone Joint Surg Am. 2005;87(1):18-27.

49. Witzleb WC, Hanisch U, Kolar N, Krummenauer F, Guenther KP. Neo-capsule tissue reactions in metal-on-metal hip arthroplasty. Acta Orthop. 2007;78(2): 211-20.

50. Clarke SA, Brooks RA, Hobby JL, Wimhurst JA, Myer BJ, Rushton N. Correlation of synovial fluid cytokine levels with histological and clinical parameters of primary and revision total hip and total knee replacements. Acta Orthop Scand. 2001;72(5):491-8.

51. Lassus J, Waris V, Xu JW, Li TF, Hao J, Nietosvaara Y, et al. Increased interleukin-8 (IL-8) expression is related to aseptic loosening of total hip replacement. Arch Orthop Trauma Surg. 2000;120(5-6):328-32.

52. Krenn V, Morawietz L, Kienapfel H, Ascherl R, Matziolis G, Hassenpflug J, et al. Revised consensus classification. Histopathological classification of diseases associated with joint endoprostheses. Z Rheumatol. 2013;72(4):383-92.

Submit your next manuscript to BioMed Central and we will help you at every step:

- We accept pre-submission inquiries

- Our selector tool helps you to find the most relevant journal

- We provide round the clock customer support

- Convenient online submission

- Thorough peer review

- Inclusion in PubMed and all major indexing services

- Maximum visibility for your research

Submit your manuscript at www.biomedcentral.com/submit
Biomed Central 